\title{
Propuesta de manejo de recursos y prioridades de investigación en el CICOLMA
}

\author{
Carlos Fragoso ${ }^{1}$
}

\section{INTRODUCCIÓN}

Sin lugar a dudas, Veracruz es uno de los estados más ricos del país, tanto por sus recursos naturales, como por sus manifestaciones culturales. En este contexto, y de acuerdo a los lineamientos del Plan Nacional de Desarrollo y el Plan Nacional de Ecología, el CICOLMA se establece como un centro cuyos principales objetivos son: 1) promover la investigación multidisciplinaria en zonas costeras, tanto básica como aplicada, con un especial énfasis sobre la relación entre ecosistemas terrestres y costeros, y 2) apoyar la enseñanza de los procesos y patrones que ocurren en estos ecosistemas, a través de cursos y seminarios.

En el CICOLMA se pretende conservar y estudiar un sector representativo de los ecosistemas costeros del estado de Veracruz, con el objeto de consolidar un espacio geográfico de trabajo para la investigación científica y tecnológica, que dé garantía de continuidad a los proyectos, a los investigadores y a las instituciones estatales y nacionales que realizen estudios en la zona.

El CICOLMA es uno más de los
centros regionales de investigación del
Instituto de Ecología. Cuenta con una
superficie de 70 ha, 48 de las cuales
corresponden a un área forestal, perteneciente
al gobierno estatal (bajo la custodia del
Centro) y 22 más propiedad del Centro, en
donde se asientan las presentes instalaciones,

así como parcelas destinadas a la investigación agropecuaria.

En esta extensión relativamente pequeña, se ha realizado y continua realizándose, una gran cantidad de investigaciones cuyos resultados podrán ser extrapolados a otras zonas costeras del estado y del país. Como ejemplo, tenemos los estudios sobre especies fijadoras de dunas, llevadas a cabo por la Dra. P. Moreno Casasola desde hace más de 10 años.

Por otro lado, el área de influencia del Centro es bastante extensa, pues incluye la cuenca hidrológica que drena parte del extremo este del Eje Neovolcánico Transverso.

\section{PROPUESTA PRELIMINAR DE MANEJO DE LOS RECURSOS DEL CENTRO}

En el manejo de los ecosistemas que se presentan en el Centro, se contemplan cuatro aspectos principales:

1) Investigaciones a realizarse

2) El papel del Centro en la formación de recursos humanos de posgrado

3) El manejo y conservación de los recursos naturales y su relación con las actividades económicas y turísticas de la región, y 4) La incidencia del Centro sobre problemas concretos que se presentan a nivel local en el aprovechamiento de los recursos naturales

1 Investigador. Departamento de Biología de Suelos. Instituto de Ecologia, A.C. Apdo. Postal 63. Xalapa, Ver., 91000 México 
(p.e. cultivos, plagas, pesca, etc.). En este documento se hara referencia a los dos últimos puntos.

\section{MANEJO Y CONSERVACION DE LOS RECURSOS NATURALES EN EL CICOLMA}

Uno de los aspectos del CICOLMA, que lo hace único en el estado de Veracruz, y quizá en el país, es su enfoque plural hacia los sistemas marinos y terrestres. En este sentido, es particularmente importante el sistema costero como la interfase mediante la cual se vinculan los procesos que ocurren en el mar y la tierra.

Por lo que respecta a los sistemas marinos y lacustres, el CICOLMA impulsará las investigaciones tendentes a optimizar el uso de los recursos pesqueros, tales como el pulpo, el ostión y una variedad de peces. También se promoverá la conservación de los sistemas arrecifales cercanos al Centro, en acciones parecidas a las que realiza actualmente el Instituto de Ecología en Isla Lobos. La conservación de las lagunetas será un aspecto prioritario, debido a su importancia como hábitat de diversas especies, entre las cuales destacan el lagarto, la iguana y numerosas aves.

Los sistemas terrestres han sido divididos en ecosistemas naturales $y$ manejados. En el primer grupo se incluyen las selvas (medianas y bajas), las dunas costeras, el manglar y el tular. En estos ecosistemas, el aspecto prioritario es la conservación y la investigación. El segundo grupo incluye pastizales y cultivos agrícolas, en donde se realizarán investigaciones aplicadas a la conservación de la fertilidad del suelo, el mejoramiento de variedades agrícolas, el mejoramiento de variedades agrícolas, el reciclaje de desechos y el mejoramiento en los sistemas de pastoreo. En el caso de las selvas, dada su pequeña extensión y por ser uno de los últimos relictos de selvas costeras sobre suelos arenosos, no se permitirá la explotación de recursos maderables, ni ningún otro tipo de manejo. Serán utilizadas intensamente en investigaciones ecológicas y taxonómicas, así como en cursos y programas de enseñanza.

En las dunas, se pondrá énfasis en el señalamiento, con objeto de conservarlas limpias y evitar la destrucción de parcelas experimentales. También serán utilizadas intensamente en investigaciones ecológicas y taxonómicas, así como en cursos y programas de enseñanza.

En el manglar y el tular, las investigaciones se concentrarán en evaluar su importancia ecológica (p.e. como sitios de nidificación) y su cambio en los últimos años. En los pastizales se realizarán investigaciones tendentes a demostrar la importancia de los árboles como focos determinantes en la dispersión de semillas y la consecuente capacidad regenerativa de los bosques tropicales. También se estudiarán y compararán métodos alternativos de pastoreo (sistema Voison) que conlleven un menor deterioro del suelo.

En los cultivos agrícolas, la investigación estará concentrada en mejorar y/o mantener la fertilidad del suelo, mediante técnicas de bajos insumos que combinen el manejo de los procesos biológicos del suelo y la aplicación de abonos verdes. También se pondrá especial atención a los problemas de plagas.

Se tiene contemplado desarrollar en las instalaciones del Centro, un proyecto de manejo de desechos orgánicos, que incluye combinar los excrementos porcinos con el cultivo de un alga, todo esto con el fin de obtener energía para el Centro.

Finalmente, existen algunas especies animales y vegetales en el Centro, que se encuentran amenazadas 0 en peligro de extinción. En estos casos se intensificará su protección, estudio y monitoreo. Entre estas especies están: diversas especies de invertebrados endémicas y exclusivas a la zona; tres especies vegetales endémicas de las dunas; el lagarto, relativamente abundante 
en la laguneta; las aves migratorias, en especial el halcón peregrino; la iguana verde; la tortuga lora, la cual se ha reportado en cinco ocasiones anidando en las playas del Centro. Aparentemente esta especie se está desplazando hacia el sur sus sitios de anidamiento, debido a la fuerte presión turística que ocurre en las playas de Texas.

Para cumplir el programa de manejo, se pretende intensificar el programa de vigilancia en el Centro, con especial énfasis en las playas y en temporadas altas de turismo. En este contexto, se tratará de sensibilizar a los turistas sobre la importancia de la zona mediante letreros y pláticas.

\section{LA INCIDENCIA DEL CICOLMA EN EL AMBITO LOCAL Y REGIONAL}

Algunas de las investigaciones que se llevan a cabo en el Centro tienen un impacto inmediato sobre las comunidades locales (fertilidad de suelos, reciclaje de desechos, pesca, etc.). En este sentido se tiene contemplado evaluar en el futuro, y de modo más objetivo, la situación ambiental. Es decir, se pretende vincular la investigación científica y el desarrollo del Cetro con la problemática socioeconómica regional, de tal manera que se logre la participación de las comunidades locales en las acciones de conservación de sus propios recursos naturales. 